\title{
Iridium-catalyzed asymmetric hydrogenation of olefins using pyridine-phosphinites derived from the chiral pool
}

\author{
Raivis Zalubovskis, ${ }^{a}$ Esther Hörmann, ${ }^{b}$ Andreas Pfaltz, ${ }^{b} *$ and Christina Moberg ${ }^{a}$ * \\ ${ }^{a}$ Department of Chemistry, KTH School of Chemical Science and Engineering, \\ SE 10044 Stockholm, Sweden \\ ${ }^{b}$ Department of Chemistry, University of Basel, St. Johanns-Ring 19, \\ CH 4056 Basel, Switzerland \\ E-mail: kimo@kth.se, andreas.pfaltz@unibas.ch
}

\begin{abstract}
Cationic iridium complexes containing chiral pyridine-phosphinites, with their chirality derived from menthol or mandelic acid, were assessed in hydrogenations of trisubstituted nonfunctionalized olefins. Complexes with the new ligands showed high reactivity towards most of the olefins tested. The enantioselectivities varied with the structures of the substrate and the ligand. Best results were obtained in the hydrogenation of 3-hydroxy-2-methyl-1-phenylpropene using a ligand prepared from (-)-menthol, pyridyllithium and diphenylchlorophosphine (>99\% conversion, $92 \%$ ee).
\end{abstract}

Keywords: Hydrogenation, iridium catalyst, chiral pool, pyridine-phosphinite, asymmetric

\section{Introduction}

Cationic iridium complexes with chiral P,N ligands have proved to be efficient catalysts for the hydrogenation of a wide range of functionalized and unfunctionalized olefins, ${ }^{1}$ Unlike Rh- and $\mathrm{Ru}$-diphosphine complexes they do not require the presence of a coordinating group near the $\mathrm{C}=\mathrm{C}$ bond, so even purely alkyl-substituted olefins can be hydrogenated with high enantioselectivity. Chiral pyridine-phosphinites (1) belong to the most efficient ligands for the catalytic process, providing highly enantioenriched products from a variety of substrates. ${ }^{2,3}$ The enantioselectivity was found to be a function of the $\mathrm{R}^{1}, \mathrm{R}^{2}$, and $\mathrm{R}^{3}$ substituents and was found to be particularly high when more rigid cyclic analogues were employed. ${ }^{3}$ The pyridinephosphinites were accessible from the appropriate chiral pyridyl alcohols, which were obtained from the racemates by preparative HPLC using a chiral column. Although this procedure gave access to the pure enantiomers, stereoselective synthesis is a more attractive option on a larger scale. Several methods are available for the synthesis of enantioenriched pyridyl alcohols, 
employing biological methods as well as synthetic reagents or catalysts. Use of hydrolases or esterases, for example, provides both enantiomers, one of them as an ester, with high enantiomeric excess. ${ }^{4,5}$ Obviously, the yield of each enantiomer is limited to a maximum of $50 \%$, which is a disadvantage if only one enantiomer is needed. In addition, not all substrates are tolerated by the enzymes. A viable alternative is the reduction of ketones using chiral reducing agents $^{2 a, 6}$ or catalysts. ${ }^{7}$ However, enantioselectivities are not perfect, so in general, the ee of the product has to be increased by recrystallization or other methods.<smiles>[R]c1cccc(C([R12])OI)n1</smiles>

An attractive alternative to these methods is based on compounds from the chiral pool. We have demonstrated that starting from cheap and easily accessible compounds such as menthol, mandelic acid, lactic acid or $(R)$-2,3-O-isopropylidene glyceraldehyde, the latter obtained by oxidative cleavage of protected mannitol, chiral stereochemically well-defined pyridyl alcohols can be obtained. ${ }^{8,9}$ We decided to evaluate pyridine-phosphinites derived from some of these pyridylalcohols in Ir-catalyzed hydrogenations of a number of unfunctionalized olefins and compare the results to those obtained using previously reported pyridine-phosphinites.

\section{Results and Discussion}

\section{Preparation of ligands}

Pyridylalcohols $\mathbf{2 a}$ and $\mathbf{2 b}$ were obtained in an 83:17 ratio (90\% yield) as previously described by $\mathrm{NaBH}_{4}$ reduction of the ketone obtained in $41 \%$ yield by reaction of 2-pyridyllithium with $(1 S, 2 S, 5 R)$-1-cyano-2-isopropyl-5-methylcyclohexane, the latter obtained from $(1 R, 2 S, 5 R)$ menthyl tosylate in $99 \%$ yield (Scheme 1). ${ }^{8,10}$ The two diastereomers were easily separated by column chromatography. Since both enantiomers of menthol are available, this procedure also gives access to the enantiomers of $\mathbf{2 a}$ and $\mathbf{2 b}$. Starting from methyl (S)-2-methoxy-2phenylacetate (methyl mandelate), an analogous procedure afforded $\mathbf{2 c}$ as a single isomer in $49 \%$ yield over two steps when the reduction was performed at low temperature $\left(-78{ }^{\circ} \mathrm{C}\right) .{ }^{11}$ The corresponding reaction with 2-bromo-6-lithiopyridine gave a ketone which was reduced in situ to a single isomer (23\% over two steps). Suzuki coupling with phenylboronic acid gave 2d $(85 \%){ }^{11}$ 


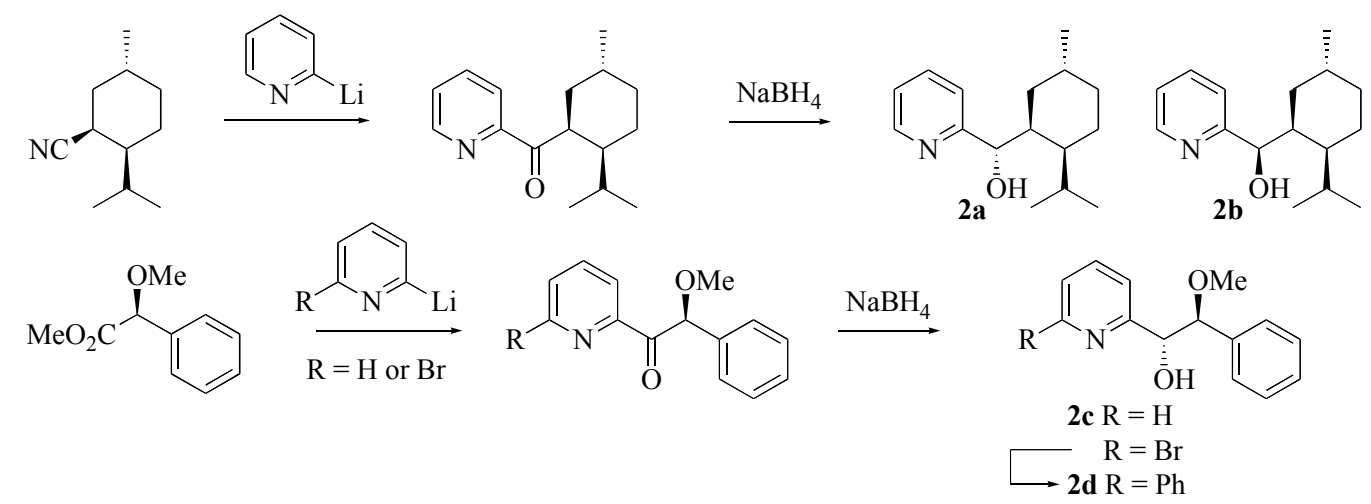

Scheme 1. Synthesis of pyridylalcohols 2a-d.

We have also demonstrated that the pyridylalcohols obtained in this way can be smoothly converted into pyridine-phosphinites. ${ }^{11}$ Thus, reactions of $\mathbf{2 a - d}$ with diphenylchlorophosphine followed by treatment with $\mathrm{BH}_{3}-\mathrm{SMe}_{2}$ proceeded efficiently, providing protected ligands 1a-d $(43 \%, 62 \%, 77 \%$ and $60 \%$, respectively). Due to the modular nature of the synthetic procedure, ligand structures are easily varied not only by employing different natural products and differently substituted pyridine derivatives, but also by using different chlorophosphines. To demonstrate this, we decided to attach a stereochemically flexible phosphepine unit to alcohols $\mathbf{2 a}$ and $\mathbf{2 b}$, hoping that the flexible unit would adopt the most suitable configuration in the catalytic reaction. Transformation of $\mathbf{2 a}$ and $\mathbf{2 b}$ to their lithium alcoholates followed by reaction of chlorophosphine $\mathbf{3}^{12}$ and $\mathrm{BH}_{3}$-protection gave access to $\mathbf{1 e}$ and $\mathbf{1}$, respectively (Scheme 2). Tropoisomerization of the phosphepine unit is expected to be slow on the NMR time scale; $\Delta \mathrm{G}^{\#}$ values for similar compounds have been determined to be around $19 \mathrm{kcal} \mathrm{mol}^{-1}$ at $298 \mathrm{~K}^{.12}$ Since the phosphorus atom is not a stereogenic center, the presence of only two diastereoisomers is thus expected. Each compound was indeed obtained as a $c a$. 1:1 mixture of the a $R$ and a $S$ diastereomers, as revealed for example by two sets of signals in their ${ }^{1} \mathrm{H}$ NMR spectra.
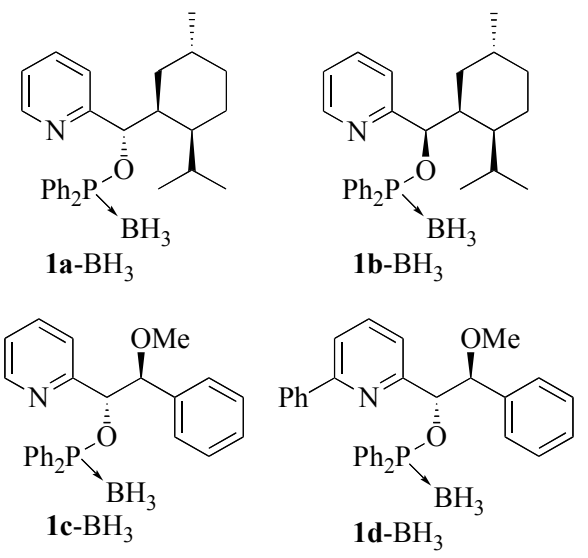

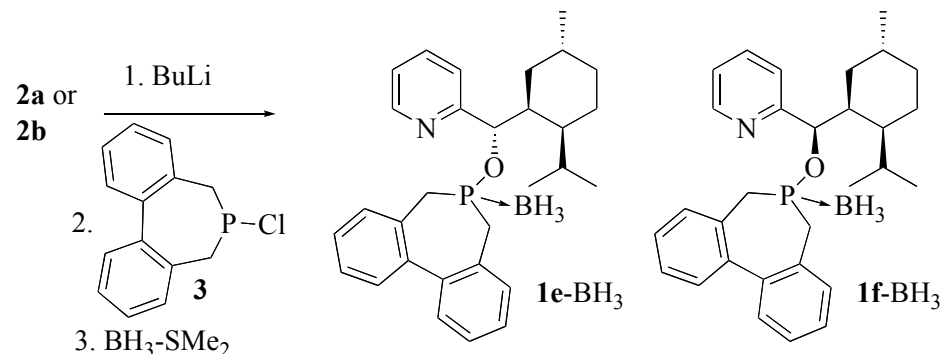

Scheme 2. Synthesis of 1e and 1f.

\section{Catalytic reactions}

Iridium complexes 4a-f were prepared by treatment of the appropriate ligand, after deprotection, with $[\operatorname{Ir}(\mathrm{COD}) \mathrm{Cl}]_{2}$ followed by sodium tetrakis[3,5-bis(trifluoromethyl)phenyl]borate. ${ }^{13}$ The complexes derived from 1e and $\mathbf{1 f}$ proved to be highly sensitive to oxygen. The complex from $\mathbf{1 f}$ was isolated in low yield after chromatography on silica-gel as an orange-red amorphous solid, whereas the analogous complex from 1e decomposed during work-up and chromatography and, therefore, was not further investigated. Catalytic hydrogenations were performed as previously described at room temperature and a hydrogen pressure of 50 bar in dichloromethane using 1 mol\% of catalyst.

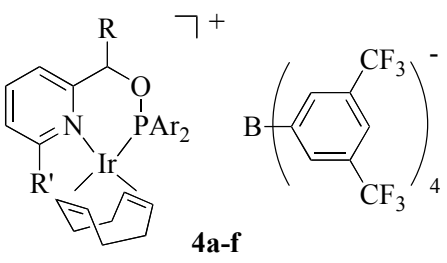

The results were compared to those previously obtained using ligands $\mathbf{1 g - 1 1}$, which contain the same type of structural elements as the new ligands $\mathbf{1 a - f .}$

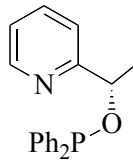

1g, $\mathrm{R}=\mathrm{Me}$

1h, $\mathrm{R}={ }^{t} \mathrm{Bu}$<smiles>POC(c1ccccc1)c1ccccn1</smiles>

$1 \mathbf{i}$

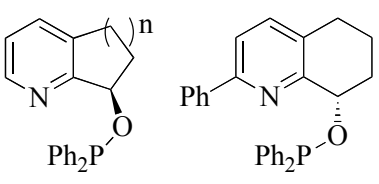

11

Trisubstituted olefins 5-10 were selected as substrates.
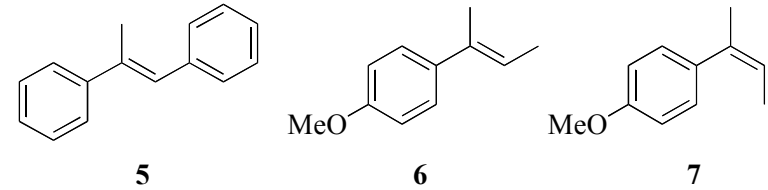

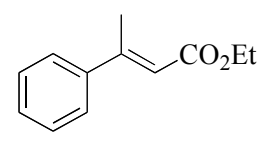

8<smiles>CC(=Cc1ccccc1)CO</smiles>

9<smiles>COc1ccc2c(c1)CCC=C2C</smiles>

10 
Table. Hydrogenation of olefins 5-10 using Ir catalysts 4a-f

\begin{tabular}{|c|c|c|c|c|c|c|}
\hline Ligand & $\begin{array}{l}\mathbf{5} \\
\text { yield }(\%) / \\
\text { ee }(\%)\end{array}$ & $\begin{array}{l}\mathbf{6} \\
\text { yield }(\%) / \\
\text { ee }(\%)\end{array}$ & $\begin{array}{l}7 \\
\text { yield }(\%) / \\
\text { ee }(\%)\end{array}$ & $\begin{array}{l}\mathbf{8} \\
\text { yield }(\%) / \\
\text { ee }(\%)\end{array}$ & $\begin{array}{l}9 \\
\text { yield }(\%) / \\
\text { ee }(\%)\end{array}$ & $\begin{array}{l}\mathbf{1 0} \\
\text { yield }(\%) / \\
\text { ee }(\%)\end{array}$ \\
\hline $1 \mathrm{a}$ & $55 / 26(S)$ & $>99 / 15(R)$ & $>99 / 60(R)$ & 21/17 (S) & $>99 / 92(+)$ & \\
\hline $1 b$ & $72 / 17(R)$ & $>99 / 19(S)$ & $>99 / 57(S)$ & $9 / 16(R)$ & $50 / 84(-)$ & $>99 / 74(S)$ \\
\hline 1c & $35 / 80(S)$ & $>99 / 65(S)$ & $97 / 56(R)$ & $3 / 0$ & $95 / 81(+)$ & $>99 / 0$ \\
\hline 1d & $0 /-$ & $46 / 55(S)$ & $17 / 75(R)$ & $1 / 12(R)$ & $5 / 14(+)$ & $12 / 34(R)$ \\
\hline 1f & $>99.7 / 24(R)$ & $>99.9 / 15(R)$ & $>99.9 / 32(S)$ & $18 / 0$ & $>99.9 / 77(-)$ & $>99.9 / 70(S)$ \\
\hline $1 \mathrm{~g}^{2 \mathrm{a}}$ & $>99 / 90(S)$ & & & & & \\
\hline $1 h^{2 a}$ & $>99 / 90(S)$ & & & & & \\
\hline $1 i^{2 \mathrm{a}}$ & $>99 / 94(R)$ & $>99 / 90(R)$ & $>99 / 87(S)$ & & & \\
\hline $1 j^{3}$ & $81 / 83(R)$ & $>99 / 78(R)$ & $>99 / 78(S)$ & $21 / 6(R)$ & $30 / 83(-)$ & $>99 / 55(S)$ \\
\hline $1 \mathrm{k}^{3}$ & $>99 / 91(R)$ & $>99 / 82(R)$ & $>99 / 83(S)$ & $24 / 26(R)$ & $26 / 89(-)$ & $>99 / 78(S)$ \\
\hline $11^{3}$ & $4 / 92(S)$ & $>99 / 96(S)$ & $>99 / 87(R)$ & $21 / 6(R)$ & $64 / 65(+)$ & $97 / 68(R)$ \\
\hline
\end{tabular}

Conditions: $\mathrm{CH}_{2} \mathrm{Cl}_{2}$, room temperature, 50 bar, $2-3 \mathrm{~h}$.

High conversions were observed in reactions of the sterically less demanding unfunctionalized olefins 6, 7, and $\mathbf{1 0}$ using all new ligands except 1d, which exhibited low reactivity, most likely as a result of steric hindrance. The $\alpha, \beta$-unsaturated ester $\mathbf{8}$ gave low conversion with all catalysts, in line with previous results reported for ligands $\mathbf{1 g}$-11. In the hydrogenation of substrates 5 and $\mathbf{9}$ large differences in reactivity were observed between catalysts 1a-1d and 1f. The enantioselectivities varied considerably with the structure of the ligand and the substrate. Methylstilbene $\mathbf{5}$ was hydrogenated efficiently only when ligand $\mathbf{1 f}$ with a phosphepine group was used, but enantioselectivities were inferior to those previously observed using ligands 1g-11. In the hydrogenation of $E$ - and $Z$-methoxystyrenes 6 and 7 the new ligands also gave lower selectivities compared to ligands 1i-11. The best results were obtained in the hydrogenation of allylic alcohol 9. Here the menthyl ligand 1a, which gave $92 \%$ ee and full conversion, clearly outperformed all other ligands, including $\mathbf{1 j} \mathbf{j} \mathbf{- 1 k}$. Finally, $\mathbf{1 b}$ and $\mathbf{1 f}$ showed results comparable to those previously observed for $1 \mathrm{k}$ and $\mathbf{1 l}$ in the hydrogenation of $\mathbf{1 0}$.

As expected, ligands 1a and $\mathbf{1 b}$ provided products with opposite absolute configuration, confirming that the stereocenter closest to phosphorus atom exerts the biggest influence on the stereoselectivity. For the same reason ligand 1c was expected to induce the same absolute configuration as 1a. This was indeed the case except for substrate 6. However, the enantioselectivities induced by ligands $\mathbf{1 a}$ and $\mathbf{1 b}$ in the hydrogenation of this substrate were very low, so these results should be interpretated with caution. Finally it may be concluded that no advantage was achieved by replacing the diphenylphosphine group by a phosphepine unit. 


\section{Conclusions}

Pyridine-phosphinites are readily available in large quantities starting with easily accessible compounds from the chiral pool. The modular approach for the ligand synthesis allows extensive structural variations since different chiral natural products, pyridine derivatives, and phosphoruscontaining groups can be assembled. Cationic iridium complexes containing the new ligands and tetrakis[3,5-bis(trifluoromethyl)phenyl]borate as counter ion were found to be reactive and moderately enantioselective catalysts in hydrogenations of several trisubstituted unfunctionalized olefins. However, quite promising results were achieved in the hydrogenation of allylic alcohol 9 which gave $92 \%$ ee and full conversion with one of the catalysts. Considering the accessibility and the modular structure of these ligands, further optimization of the catalyst structure should be possible.

\section{Experimental Section}

General Procedures. ${ }^{1} \mathrm{H}$ NMR spectra were recorded at 400 or $500 \mathrm{MHz},{ }^{13} \mathrm{C}$ NMR at 125 or $100 \mathrm{MHz}$ and ${ }^{31} \mathrm{P}$ NMR at $202 \mathrm{MHz}$. The ${ }^{1} \mathrm{H}$ and ${ }^{13} \mathrm{C}$ chemical shifts are reported relative to remaining solvent peaks $\left(\mathrm{CHCl}_{3}\right),{ }^{31} \mathrm{P}$ chemical shifts are reported relative to $\mathrm{H}_{3} \mathrm{PO}_{4}$ (external). Optical rotations were recorded with a Perkin-Elmer 343 polarimeter at the sodium D line at ambient temperature. Air sensitive reactions were performed under nitrogen atmosphere in ovenor flame-dried glassware. $\mathrm{CH}_{2} \mathrm{Cl}_{2}$, PhMe and THF were taken from Meyer's Solvent Dispensing System. Flash chromatography was carried out using SDS silica gel $60(40-63 \mu \mathrm{m})$.

\section{Compound 1e- $\mathrm{BH}_{3}$}

$n$-BuLi $(236 \mu \mathrm{L}, 1.40 \mathrm{M}, 0.33 \mathrm{mmol})$ was added to a degassed solution of alcohol $2 \mathbf{a}(80 \mathrm{mg}$, $0.32 \mathrm{mmol})$ in THF $(2 \mathrm{~mL})$ at $-78^{\circ} \mathrm{C}$ and the mixture was stirred at this temperature for $30 \mathrm{~min}$. The temperature was increased to $0{ }^{\circ} \mathrm{C}$ and a degassed solution of phosphochloride 3 ( $86 \mathrm{mg}$, $0.35 \mathrm{mmol})$ in THF $(0.7 \mathrm{~mL})$ was added. The mixture was stirred at $0{ }^{\circ} \mathrm{C}$ for $3 \mathrm{~h}$ before $\mathrm{BH}_{3} \cdot \mathrm{Me}_{2} \mathrm{~S}(175 \mu \mathrm{L}, 2 \mathrm{M}$ in THF, $0.35 \mathrm{mmol})$ was added and the resulting mixture was stirred at 0 ${ }^{\circ} \mathrm{C}$ for $14 \mathrm{~h} . \mathrm{H}_{2} \mathrm{O}(5 \mathrm{~mL})$ was added and the solvent was evaporated under vacuum. The crude product was purified by column chromatography on silica gel $\left(\mathrm{CH}_{2} \mathrm{Cl}_{2} / \mathrm{EtOAc} 98: 2\right)$ to give 1e$\mathrm{BH}_{3}$ (70 mg, 46\%); $[\alpha]_{\mathrm{D}}^{25}+17.9\left(c 0.50, \mathrm{CH}_{2} \mathrm{Cl}_{2}\right) ;{ }^{1} \mathrm{H} \mathrm{NMR}\left(500 \mathrm{MHz}, \mathrm{CDCl}_{3}\right.$ ) (mixture of two atropisomers) $\delta 8.88$ (br d, $J=4.7 \mathrm{~Hz}, 1 \mathrm{H}), 8.53(\mathrm{br} \mathrm{d}, J=4.7 \mathrm{~Hz}, 1 \mathrm{H}), 7.81(\mathrm{dt}, J=7.6,1.8 \mathrm{~Hz}$, $1 \mathrm{H}), 7.59(\mathrm{dt}, J=7.7,1.7 \mathrm{~Hz}, 1 \mathrm{H}), 7.50(\mathrm{~d}, J=7.8 \mathrm{~Hz}, 1 \mathrm{H}), 7.41-7.14(\mathrm{~m}, 17 \mathrm{H}), 7.05(\mathrm{~d}, J=7.6$ $\mathrm{Hz}, 1 \mathrm{H}), 6.54(\mathrm{~d}, J=7.6 \mathrm{~Hz}, 1 \mathrm{H}), 5.70-5.63(\mathrm{~m}, 2 \times 1 \mathrm{H}), 3.11-2.76(\mathrm{~m}, 5 \mathrm{H}), 2.73-2.67(\mathrm{~m}, 1 \mathrm{H})$, $2.42(\mathrm{~d}, J=15.2 \mathrm{~Hz}, 1 \mathrm{H}), 2.22-2.13(\mathrm{~m}, 2 \mathrm{H}), 2.08-1.98(\mathrm{~m}, 3 \mathrm{H}), 1.95-1.80(\mathrm{~m}, 4 \mathrm{H}), 1.74-1.53$ $(\mathrm{m}, 6 \mathrm{H}), 1.53-1.42(\mathrm{~m}, 1 \mathrm{H}), 1.29-1.06(\mathrm{~m}, 3 \mathrm{H}), 1.25(\mathrm{~d}, J=6.5 \mathrm{~Hz}, 3 \mathrm{H}), 1.02(\mathrm{~d}, J=6.5 \mathrm{~Hz}$, $3 \mathrm{H}), 1.0-0.2$ (br, $2 \times 3 \mathrm{H}), 0.95-0.81(\mathrm{~m}, 2 \mathrm{H}), 0.92$ (d, $J=6.4 \mathrm{~Hz}, 3 \mathrm{H}), 0.89$ (d, $J=6.4 \mathrm{~Hz}, 3 \mathrm{H})$, $0.60(\mathrm{~d}, J=6.5 \mathrm{~Hz}, 3 \mathrm{H}), 0.58(\mathrm{~d}, J=6.5 \mathrm{~Hz}, 3 \mathrm{H}) ;{ }^{13} \mathrm{C} \mathrm{NMR}\left(125 \mathrm{MHz}, \mathrm{CDCl}_{3}\right) \delta 159.9,159.7$, 
$150.1,149.4,140.4,140.1,140.0,139.8\left(\mathrm{~d}, J_{\mathrm{CP}}=2.8 \mathrm{~Hz}\right), 139.5\left(\mathrm{~d}, J_{\mathrm{CP}}=2.7 \mathrm{~Hz}\right), 136.6,136.4$, 131.6, 131.5, 131.3, 131.2, $131.0\left(\mathrm{~d}, J_{\mathrm{CP}}=3.6 \mathrm{~Hz}\right), 130.6,130.5,130.4,130.3,130.2,130.1$, $130.0\left(\mathrm{~d}, J_{\mathrm{CP}}=3.8 \mathrm{~Hz}\right), 129.9\left(\mathrm{~d}, J_{\mathrm{CP}}=3.8 \mathrm{~Hz}\right), 129.5,129.4,129.3,128.0\left(\mathrm{~d}, J_{\mathrm{CP}}=1.8 \mathrm{~Hz}\right)$, $127.8,127.7\left(\mathrm{~d}, J_{\mathrm{CP}}=2.7 \mathrm{~Hz}\right), 127.6\left(\mathrm{~d}, J_{\mathrm{CP}}=2.1 \mathrm{~Hz}\right), 127.3\left(\mathrm{~d}, J_{\mathrm{CP}}=2.1 \mathrm{~Hz}\right), 124.1,123.5$, $123.2\left(\mathrm{~d}, J_{\mathrm{CP}}=3.2 \mathrm{~Hz}\right), 81.3\left(\mathrm{~d}, J_{\mathrm{CP}}=4.0 \mathrm{~Hz}\right), 81.2\left(\mathrm{~d}, J_{\mathrm{CP}}=4.0 \mathrm{~Hz}\right), 50.0,40.5\left(\mathrm{~d}, J_{\mathrm{CP}}=7.0 \mathrm{~Hz}\right)$, $39.8\left(\mathrm{~d}, J_{\mathrm{CP}}=7.0 \mathrm{~Hz}\right), 38.7,38.5,35.9,35.8,34.6\left(\mathrm{~d}, J_{\mathrm{CP}}=28.0 \mathrm{~Hz}\right), 34.1\left(\mathrm{~d}, J_{\mathrm{CP}}=28.1 \mathrm{~Hz}\right)$, 34.0, 33.8, 33.7, 33.5, 33.4, 30.3, 29.9, 26.8, 26.7, 24.9, 24.7, 23.0, 22.7 (d, $\left.J_{\mathrm{CP}}=11.5 \mathrm{~Hz}\right), 22.6$ $\left(\mathrm{d}, J_{\mathrm{CP}}=12.7 \mathrm{~Hz}\right), 22.1 ;{ }^{31} \mathrm{P} \mathrm{NMR}\left(202 \mathrm{MHz}, \mathrm{CDCl}_{3}\right) \delta 144.0(\mathrm{br}), 142.7$ (br); Anal. Calcd for $\mathrm{C}_{30} \mathrm{H}_{39}$ BNOP: C, 76.43; H, 8.34; N, 2.97\% Found: C, 76.27; H, 8.30; N, 2.78\%

\section{Compound 1f- $\mathrm{BH}_{3}$}

This compound was prepared from alcohol $\mathbf{2 b}(61 \mathrm{mg}, 0.25 \mathrm{mmol})$ and phosphochloride 3 (67 $\mathrm{mg}, 0.27 \mathrm{mmol}$ ), by the same procedure as that used for $1 \mathrm{e}$ (eluent for chromatography: $\mathrm{CH}_{2} \mathrm{Cl}_{2}$ ). Yield 35\% (41 mg); $[\alpha]_{\mathrm{D}}^{25}-30.0\left(c 0.50, \mathrm{CH}_{2} \mathrm{Cl}_{2}\right) ;{ }^{1} \mathrm{H}$ NMR $\left(500 \mathrm{MHz}, \mathrm{CDCl}_{3}\right)$ (mixture of two atropisomers) $\delta 8.77$ (br d, $J=4.8 \mathrm{~Hz}, 1 \mathrm{H}), 8.54(\mathrm{~d}, J=4.9 \mathrm{~Hz}, 1 \mathrm{H}), 7.76(\mathrm{dt}, J=7.7,1.5 \mathrm{~Hz}$, $1 \mathrm{H}), 7.58(\mathrm{dt}, J=7.7,1.6 \mathrm{~Hz}, 1 \mathrm{H}), 7.48-7.21(\mathrm{~m}, 17 \mathrm{H}), 7.17-7.09(\mathrm{~m}, 2 \mathrm{H}), 6.91(\mathrm{~d}, J=7.6 \mathrm{~Hz}$, $1 \mathrm{H}), 5.68-5.58(\mathrm{~m}, 2 \times 1 \mathrm{H}), 3.13-2.84(\mathrm{~m}, 4 \mathrm{H}), 2.74-2.43(\mathrm{~m}, 6 \mathrm{H}), 2.00-1.55(\mathrm{~m}, 10 \mathrm{H}), 1.50-1.40$ $(\mathrm{m}, 1 \mathrm{H}), 1.35-1.25(\mathrm{~m}, 1 \mathrm{H}), 1.25-1.15(\mathrm{~m}, 1 \mathrm{H}), 1.14-0.80(\mathrm{~m}, 5 \mathrm{H}), 0.90(\mathrm{~d}, J=6.0 \mathrm{~Hz}, 3 \mathrm{H}), 0.89$ $(\mathrm{d}, J=6.4 \mathrm{~Hz}, 3 \mathrm{H})$, ca $0.8-0.15(\mathrm{br}, 2 \times 3 \mathrm{H}), 0.81(\mathrm{~d}, J=6.0 \mathrm{~Hz}, 3 \mathrm{H}), 0.80(\mathrm{~d}, J=6.0 \mathrm{~Hz}, 3 \mathrm{H})$, $0.74(\mathrm{~d}, J=6.4 \mathrm{~Hz}, 3 \mathrm{H}), 0.64(\mathrm{~d}, J=6.4 \mathrm{~Hz}, 3 \mathrm{H}) ;{ }^{13} \mathrm{C} \mathrm{NMR}\left(125 \mathrm{MHz}, \mathrm{CDCl}_{3}\right) \delta 161.0,149.4$, $149.2,140.2,140.1,139.8\left(\mathrm{~d}, J_{\mathrm{CP}}=2.6 \mathrm{~Hz}\right), 139.6\left(\mathrm{~d}, J_{\mathrm{CP}}=2.7 \mathrm{~Hz}\right), 136.3,135.9,131.7,131.6$ $\left(\mathrm{d}, J_{\mathrm{CP}}=2.5 \mathrm{~Hz}\right), 131.5,131.2\left(\mathrm{~d}, J_{\mathrm{CP}}=4.0 \mathrm{~Hz}\right), 130.7\left(\mathrm{~d}, J_{\mathrm{CP}}=3.8 \mathrm{~Hz}\right), 130.5,130.4,130.3$, $130.2,130.0\left(\mathrm{~d}, J_{\mathrm{CP}}=3.1 \mathrm{~Hz}\right), 129.8\left(\mathrm{~d}, J_{\mathrm{CP}}=3.4 \mathrm{~Hz}\right), 129.6,129.4,129.3\left(\mathrm{~d}, J_{\mathrm{CP}}=2.0 \mathrm{~Hz}\right)$, $129.2\left(\mathrm{~d}, J_{\mathrm{CP}}=2.0 \mathrm{~Hz}\right), 128.0\left(\mathrm{~d}, J_{\mathrm{CP}}=1.8 \mathrm{~Hz}\right), 127.9\left(\mathrm{~d}, J_{\mathrm{CP}}=1.8 \mathrm{~Hz}\right), 127.8,127.7,127.6(\mathrm{~d}$, $\left.J_{\mathrm{CP}}=3.0 \mathrm{~Hz}\right), 127.5,127.4\left(\mathrm{~d}, J_{\mathrm{CP}}=2.4 \mathrm{~Hz}\right), 123.0,122.9,122.7,81.3\left(\mathrm{~d}, J_{\mathrm{CP}}=4.5 \mathrm{~Hz}\right), 81.0(\mathrm{~d}$, $\left.J_{\mathrm{CP}}=4.5 \mathrm{~Hz}\right), 48.4,48.3,40.0\left(\mathrm{~d}, J_{\mathrm{CP}}=6.6 \mathrm{~Hz}\right), 39.8\left(\mathrm{~d}, J_{\mathrm{CP}}=6.5 \mathrm{~Hz}\right), 36.6,36.3,35.9,35.6$, 35.4, 35.2, 34.6, 34.5, 34.4, 34.3, 34.2, 29.9 (d, $\left.J_{\mathrm{CP}}=21.0 \mathrm{~Hz}\right), 27.3\left(\mathrm{~d}, J_{\mathrm{CP}}=7.7 \mathrm{~Hz}\right), 25.9,25.6$, 23.2, 22.9, $21.8\left(\mathrm{~d}, J_{\mathrm{CP}}=17.3 \mathrm{~Hz}\right), 21.2\left(\mathrm{~d}, J_{\mathrm{CP}}=12.5 \mathrm{~Hz}\right) ;{ }^{31} \mathrm{P}$ NMR $\left(202 \mathrm{MHz}, \mathrm{CDCl}_{3}\right) \delta 145.3$ (br); Anal. Calcd for $\mathrm{C}_{30} \mathrm{H}_{39}$ BNOP: C, 76.43; H, 8.34; N, 2.97\% Found: C, 76.19; H, 8.38; N, $2.70 \%$

\section{Complex 4f}

1f $-\mathrm{BH}_{3}(50 \mathrm{mg}, 0.1 \mathrm{mmol})$ was deprotected using diethylamine $(2 \mathrm{~mL})$. Ligand $\mathbf{1 f}$ was dissolved in $\mathrm{CH}_{2} \mathrm{Cl}_{2}(4 \mathrm{~mL})$, and added to $[\mathrm{Ir}(\mathrm{COD}) \mathrm{Cl}]_{2}(37 \mathrm{mg}, 0.55 \mathrm{mmol})$ in $\mathrm{CH}_{2} \mathrm{Cl}_{2}(1 \mathrm{~mL})$, to give an orange solution which was stirred at $50{ }^{\circ} \mathrm{C}$ for $2 \mathrm{~h}$. After cooling to room temperature, $\mathrm{NaBAr}_{\mathrm{F}} \mathrm{x} 3 \mathrm{H}_{2} \mathrm{O}(122 \mathrm{mg}, 1.3 \mathrm{mmol})$ was added and the resulting red-orange mixture was stirred overnight. Then $\mathrm{H}_{2} \mathrm{O}(2 \mathrm{~mL})$ was added and the mixture stirred for a further $30 \mathrm{~min}$. The organic solvent was removed, the water phase extracted with $\mathrm{CH}_{2} \mathrm{Cl}_{2}(2 \times 3 \mathrm{~mL})$ and the collected organic solution dried over $\mathrm{MgSO}_{4}$. The solvent was removed under vacuum at room temperature to give a brownish-red solid. The crude product was purified under $\mathrm{N}_{2}$ by flash chromatography on silica 
gel using $\mathrm{Et}_{2} \mathrm{O}$ and $\mathrm{CH}_{2} \mathrm{Cl}_{2}$ as eluents (gradient mixture, $\mathrm{CH}_{2} \mathrm{Cl}_{2}$ previously stirred over basic aluminum oxide for $30 \mathrm{~min}$ ) and the oily solid obtained was washed with pentane to yield a redorange solid (35 mg, 21\%). The ESI-MS showed the expected signal group of complex $\mathbf{4 f}$ at $\mathrm{m} / \mathrm{z}$ $756\left(61 \% ;{ }^{191} \mathrm{Ir}\right), 757(24 \%), 758\left(100 \% ;{ }^{193} \mathrm{Ir}\right), 759(38 \%)$ and $760(8 \%)$; no other peaks with intensities $>3 \%$ were detected.

\section{Acknowledgements}

This work was supported by the Swedish Research Council and the Swiss National Science Foundation.

\section{References}

1. Pfaltz, A.; Blankenstein, J.; Hilgraf, R.; Hörmann, E.; McIntyre, S.; Menges, F.; Schönleber, M.; Smidt, S. P.; Wüstenberg, B.; Zimmermann, N. Adv. Synth. Catal. 2003, 345, 33. (b) Cui, X.; Burgess, K. Chem. Rev. 2005, 105, 3272. (c) Källström, K.; Munslow, I.; Andersson, P. G. Chem. Eur. J. 2006, 12, 3194. (d) Roseblade, S. J.; Pfaltz, A. Acc. Chem. Res. 2007, 40, 1402.

2. Drury III, W. J.; Zimmermann, N.; Keenan, M.; Hayashi, M.; Kaiser, S.; Goddard, R.; Pfaltz, A. Angew. Chem. Int. Ed. 2004, 43, 70. (b) Bell, S.; Wüstenberg, B.; Kaiser, S.; Menges, F.; Netscher, T.; Pfaltz, A. Science 2006, 311, 642. (c) Wang, A.; Wüstenberg, B.; Pfaltz, A. Angew. Chem. Int. Ed. 2008, 47, 2298.

3. Kaiser, S.; Smidt, S. P.; Pfaltz, A. Angew. Chem. Int. Ed. 2006, 45, 5194.

4. Uenishi, J.; Hamada, M. Synthesis 2002, 625.

5. For the kinetic resolution of pyridyl alcohols using $\mathrm{Cu}(\mathrm{II})$-bisoxazoline acylation catalysts, see: Mazet, C.; Roseblade, S.; Köhler, V.; Pfaltz, A. Org. Lett. 2006, 9, 1879.

6. Bolm, C.; Ewald, M.; Felder, M.; Schlingloff, G. Chem. Ber. 1992, 125, 1169.

7. (a)Ohkuma, T.; Koizumi, M.; Makatao, Y.; Noyori, R. Org. Lett. 2000, 2, 1749. (b) Okano, K.; Murata, K.; Ikariya, T. Tetrahedron Lett. 2000, 41, 9277. (c) Lipshutz, B.; Lower, A.; Noson, K. Org. Lett. 2002, 4, 4045. (d) Corey, E. J.; Helal, C. J. Tetrahedron Lett. 1996, 37, 5675 .

8. Adolfsson, H.; Nordström, K.; Wärnmark, K.; Moberg, C. Tetrahedron: Asymmetry 1996, 7, 1967.

9. Rahm, F.; Stranne, R.; Bremberg, U.; Nordström, K.; Cernerud, M.; Macedo, E.; Moberg, C. J. Chem. Soc., Perkin. Trans. 1 2000, 1983.

10. Correction of the determination of absolute stereochemistry of the pyridine alcohols: Cunningham, D.; Gallagher, E. T.; Grayson, D. H.; McArdle, P. J.; Storey, C. B.; Wilcock, D. J. J. Chem. Soc., Perkin Trans. 1 2002, 2692. 
11. Rahm, F.; Fischer, A.; Moberg, C. Eur. J. Org. Chem. 2003, 4205.

12. Zalubovskis, R.; Fjellander, E.; Szabo, Z.; Moberg, C. Eur. J. Org. Chem. 2007, 108.

13. Lightfoot, A.; Schnider, P.; Pfaltz, A. Angew. Chem. Int. Ed. 1998, 372897. 